\title{
Estudo das perdas de água no sistema de abastecimento da cidade de Porto Nacional/TO
}

As perdas em sistema de abastecimento de água das zonas urbanas são vistas como um grande problema que as concessionárias de saneamento tem que lida cotidianamente, pois o crescimento continuo dos centros urbanos de forma desordenada, reflete no problema da má infraestrutura, expansão dos sistemas de abastecimento e na instalação de novas fontes de distribuição de água tratada, bem como a deterioração dos sistemas já existentes ao logo dos anos de utilização. Esses fatores mencionados unidos entre si, implicam em um grande volume de perdas, através de altas pressões dentro da rede ou por meio de vazamentos. Em alguns casos específicos as perdas são apresentadas também com casos de fraudes, feitas pela população e de forma irregular, ou mesmo falha dos medidores, que também entram na contagem de perdas. Desse modo, o presente trabalho propõe uma análise do volume de perdas da cidade de Porto Nacional/TO, bem como as principais soluções e medidas que estão sendo tomadas para que haja um melhor desempenho da distribuição de água.

Palavras-chave: Perdas; Sistema de Abastecimento; Distribuição de Água.

\section{Study of water losses in the supply system of the city of Porto Nacional/TO}

\begin{abstract}
Losses in water supply systems in urban areas are seen as a major problem that sanitation utilities have to deal with on a daily basis, as the continued growth of urban centers in a disorderly manner, reflects on the problem of poor infrastructure, expansion of supply systems and the installation of new sources of treated water distribution, as well as the deterioration of existing systems over the years of use. These factors mentioned together, imply a large volume of losses, through high pressures within the network or through leaks. In some specific cases the losses are also presented with cases of fraud, made by the populations and in an irregular way, or even failure of the meters, which also count in the loss count. Thus, the present work proposes a demonstrative analysis of the volume of losses in the city of Porto Nacional/TO, as well as the main solutions and measures that are Bering taken so that there is a better performance of water distribution.
\end{abstract}

Keywords: Losses; Supply system; Water distribution.

Topic: Engenharia Sanitária

Reviewed anonymously in the process of blind peer.
Received: 09/07/2020

Approved: 22/10/2020
Pedro Henrique Souza Barros (iD

Faculdade Presidente Antônio Carlos, Brasi http://lattes.cnpq.br/2347931152168053 http://orcid.org/0000-0003-4830-4348

pedro.engcivil22.barros@gmail.com

Diogo Pedreira Lima

Faculdade Presidente Antônio Carlos, Brasil http://lattes.cnpq.br/7967728577417186 http://orcid.org/0000-0002-3849-2587

diogo.lima@itpacporto.edu.br
Referencing this:

BARROS, P. H. S.; LIMA, D. P.. Estudo das perdas de água no sistema de abastecimento da cidade de Porto Nacional/TO. Natural Resources, v.10, n.3, p.103-112, 2020. DOI:

http://doi.org/10.6008/CBPC2237-9290.2020.003.0011 


\section{INTRODUÇÃO}

Em todas as épocas, o abastecimento de água para uso e consumo humano sempre foi uma preocupação dentre todos os povos. Desde de os primórdios, as civilizações se concentravam em construir suas casas e fazer plantações as margens dos cursos de água, pois sem água a vida humana existente seria extinta, visto que 70 \% da composição do corpo humano é constituído por água. Fazendo a análise histórica de alguns milhares de anos atrás, em diversos documentos e registros são atestados a grande preocupação que o homem sempre teve em abastecer de água suas comunidades. Tendo como grande exemplo, a Roma Antiga, que para abastecer a população daquela época, desenvolveu uma variedade de obras hidráulicas para distribuição da água (CREDER, 2006).

As Companhias de Abastecimento de Água têm como um dos principais desafios a aplicação constante de novas tecnologias e práticas que garante uma maior racionalização dos recursos hídricos e também controle de perdas (GONÇALVES, 2018). O sistema de abastecimento e distribuição de água é considerado um conjunto de processos formados por partes. Sendo que cada parte em específico possui sua função e/ou objetivo, correspondendo aos órgãos compostos por obras presentes na construção civil, equipamentos elétricos e eletrônicos, acessórios, instrumentação e equipamentos de automação e controle (SOUSA, 2001).

Em um sistema de abastecimento de água, o volume de perdas é considerado como o motivo principal para determinação da eficiência nas tarefas comerciais e de distribuição. Ao realizar-se uma análise sobre a situação atual de perdas em uns sistemas de abastecimento, é levado em consideração o índice existente de água desperdiçada, ou seja, à medida que os níveis de desperdício aumentam, surgem também uma maior necessidade e empenho de esforços, que atuaram na redução de possíveis ineficiências entre as fases de manutenção, planejamento, rota de investimentos e ocupações ligadas a operação e comércio (TRATA BRASIL, 2019).

No que diz respeito a perdas de água, em sua grande maioria são causadas por uma deficiência nas tubulações em razão do baixo índice de manutenção e operação, bem como uma inadequada gestão comercial por parte das concessionaria local. Porém, atualmente, existe uma variedade altíssima no índice de perdas, onde em alguns casos chegam a atingir $60 \%$ dentre o valor total de água tratada disponibilizada, e com isso as empresas de saneamento tendem a criar um modelo de programa com intuito de reduzir o índice das perdas, tendo em vista um melhor desempenho econômico das empresas e uma melhor imagem da companhia em relação ao cliente (TSUTIYA, 2005).

O abastecimento de água em certas atividades econômicas, como zonas agrícolas, apresenta um grau de perda produtiva, em vista de sua maior complexibilidade e características distintas, sendo assim, para esses setores a ideia de perda consiste em ser reduzida a zero. Todavia, o Brasil precisa restituir-se de métodos que possam evitar o continuo desperdício da água tratada, além de aperfeiçoar má gestão de suas empresas e serviços de saneamento (WERDINE, 2002).

O problema das perdas é constituído em uma escala mundial, acarretando em um baixo desempenho 
em sua grande maioria dos sistemas de abastecimento. Contudo, existem exceções onde países e cidades, com conhecimento, planejamento, recursos e gestão conseguiram alcançar e permanecer com baixos volumes de perda dentro dos seus sistemas (ABES, 2015).

No Brasil, a estadia que garante a implementação de novos métodos e ações para o combate efetivos das perdas, apresenta-se ainda maior devido ao grande volume recorrente de déficits hídrico dentre as diferentes zonas do país. As cidades que possuem maior padrão de notoriedade em perdas apresentam uma média de 15\% em seus indicadores. Em 2016, no brasil foram registrados um índice com relação a perdas em zonas de distribuição de $38,05 \%$, sendo que o índice de perdas faturado totalizou em $38,85 \%$, fator esse que torna um percurso bem longo a ser seguido para resolução de maior eficácia (TRATA BRASIL, 2019).

O presente trabalho pretende relatar uma abordagem de perdas no abastecimento de água da cidade de Porto Nacional, Tocantins, visando uma avaliação do panorama em relação ao quantitativo geral das perdas, proporcionando em sequência um melhor planejamento para o controle e redução do desperdício de água na cidade.

\section{METODOLOGIA}

Através de dados obtidos na concessionária local e contidos no Plano Municipal de Água e esgoto (PMAE/PN-TO), o presente trabalho analisou minuciosamente alguns parâmetros pertinentes as perdas de água geradas no município, os dados utilizados estão datados entre o período de Abril de 2016 a Março de 2017.

\section{Área de Estudo}

A área de estudo escolhida para realização desse trabalho foi a cidade de Porto Nacional/TO, situada numa região as margens do Rio Tocantins. O município de Porto Nacional possui as seguintes características geográficas: 4 449,917 km² de área; 52700 habitantes; densidade demográfica de 11,8 habitantes por km². Está situada ao leste da capital do Estado Palmas, cerca de $60 \mathrm{Km}$ de distância. Possui relevo plano, estando a uma altitude equivalente de 212 metros, e suas coordenadas geográficas são Latitude: 1042'28" Sul e longitude 48이'01" Oeste.

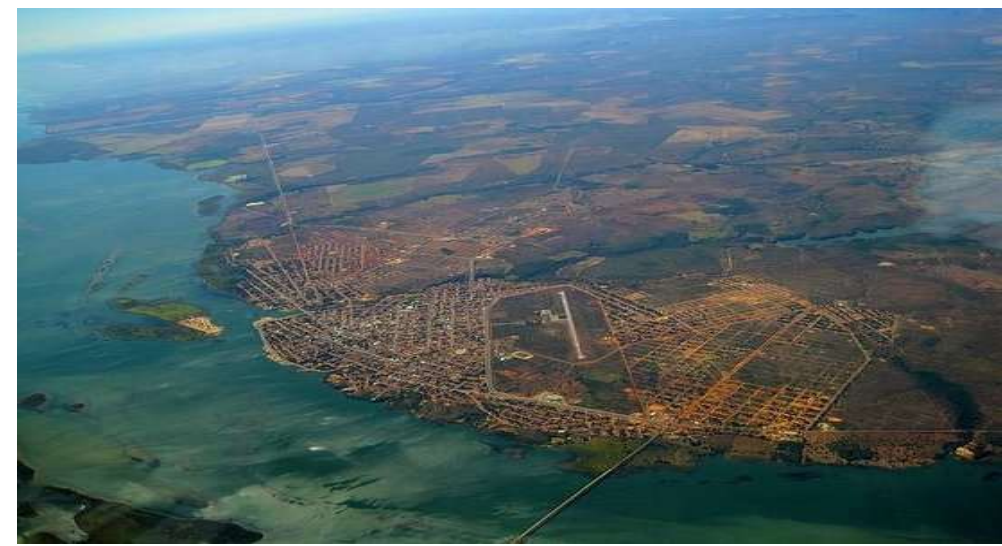

Figura 1: Vista Aérea da zona urbana na cidade de Porto Nacional/TO. 
Com idade de 157 anos desde sua emancipação e contexto histórico que de 280 anos, a cidade possui uns vastos sistemas de distribuição de água tratada, que garante atendimento de $99,9 \%$ da população. A figura 1 abaixo, apresenta uma visão geral do território urbano da cidade.

\section{Contexto Histórico do Saneamento da Cidade}

De acordo com o Plano Municipal de Água e Esgoto PMAE/PN-TO, revisado em 2018, a princípio o abastecimento de água da cidade de Porto Nacional, era conduzido interinamente pela Companhia de Saneamento de Goiás (SANEAGO), porém após o desmembramento entre estados, em 1998, foi criada a SANEATINS - Companhia de Saneamento do Tocantins, que ficou a partir de então responsável pelo desenvolvimento dos serviços de saneamento do novo Estado. Em janeiro de 2012, o governo do Estado do Tocantins, optou por transformar a SANEATINS uma empresa independente, através da iniciativa privada, passando assim a ser gerenciada pela Odebrecht Ambiental.

Atualmente, o gerenciamento da área de saneamento da cidade de Porto Nacional está sobre jurisdição da BRK Ambiental, que assumiu o controle em maio de 2017, tendo em seu status atual o cargo de maior empresa privada de saneamento do país. Além disso, faz parte do Grupo Brookfield, empresa canadense que investe e administra ativos em mais de 30 países, dentro dos 5 continentes, e chegou ao Brasil em 1899. De modo a garantir um o desenvolvimento social, de uma área importante para o futuro do país e sua população, a BRK Ambiental assegura uma parceria minoritária com o FI FGTS (Fundo de Investimentos do Fundo de Garantia Por Tempo de Serviço).

\section{RESULTADOS}

O Plano Municipal de Água e Esgoto (PMAE/PN-TO,2018), retrata que a captação da água na Sede do município de Porto Nacional é feita no Ribeirão São João, rodeado por uma barragem para garantir o acumulo de água. A partir daí a água bruta é coletada e bombeada para a Estação de Tratamento de Água (ETA-002), localizada na Rua Contorno, $\mathrm{s} / \mathrm{n}^{\circ}$, Setor Parque Eldorado onde passa a ser devidamente tratada e em seguida distribuída. A ETA-002 em sua composição para tratamento da água, possui um sistema de filtração duplo, que garante uma vazão média de $612 \mathrm{~m}^{3} / \mathrm{h}$ (2018), operando num período entre 18 e 21 horas diárias. A reservação total em operação da cidade é de $4.025 \mathrm{~m}^{3}$.

As unidades citadas abaixo, representam os componentes do sistema de abastecimento atual do município: Captação de Água Superficial no Córrego São João; Elevatórias de água Bruta - EEAB; Adutora de água Bruta; Estação de Tratamento de Água; Elevatórias de água tratada - EEAT; Adutoras de água tratada; 05 Reservatórios; Escritório; Redes de Distribuição.

A Sede de Porto Nacional, possui um sistema de abastecimento de água que atende toda a zona urbana. No mapa abaixo, encontra-se os sistemas anteriormente citados e também a localização dos bairros atendidos: 


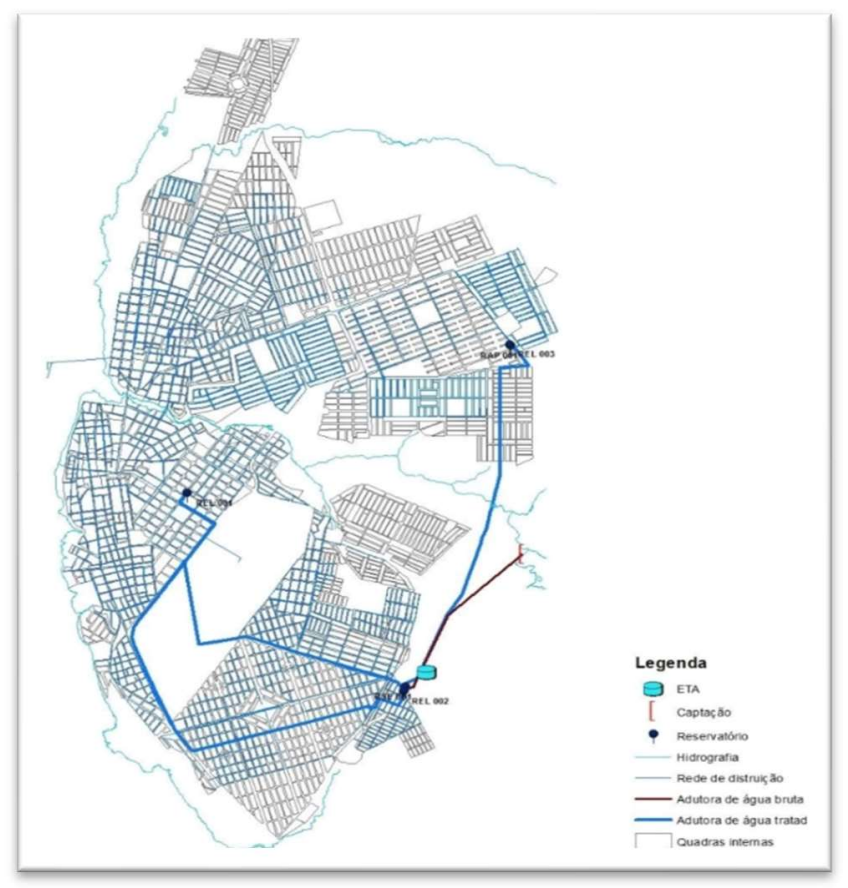

Figura 2: Atendimento de água de Porto Nacional/TO.

O quadro 1 também especifica as informações pertinentes aos sistemas de reservação citados anteriormente.

Quadro 1: Característica dos reservatórios da cidade de Porto Nacional/TO.

\begin{tabular}{|l|l|l|l|l|l|}
\hline RESERVATÓRIO & CAPACIDADE (M $\left.\mathbf{M}^{\mathbf{3}}\right)$ & TIPO & FUNÇÃO PRINCIPAL & MATERIAL & LOCALIZAÇÃO \\
\hline RSE 01 & 2.700 & Semienterrado & Distribuição & Concreto & ETA 002 \\
\hline REL 02 & 125 & Elevado & Distribuição & Concreto & ETA 002 \\
\hline REL 01 & 125 & Elevado & Distribuição & Concreto & Adm. Central \\
\hline RAP 01 & 1.000 & Apoiado & Distribuição & Metálico & Setor Ypê \\
\hline REL 03 & 75 & Elevado & Distribuição & Metálico & Setor Ypê \\
\hline TOTAL & $\mathbf{4 . 0 2 5}$ & & & & \\
\hline
\end{tabular}

Fonte: Concessionária

\section{Volume Produzido de água}

O gráfico da figura abaixo, foi elaborado para obter-se uma visualização melhor do volume disponibilizado, sendo os dados apresentados referente ao período entre Abr/2016 e Mar/2017.

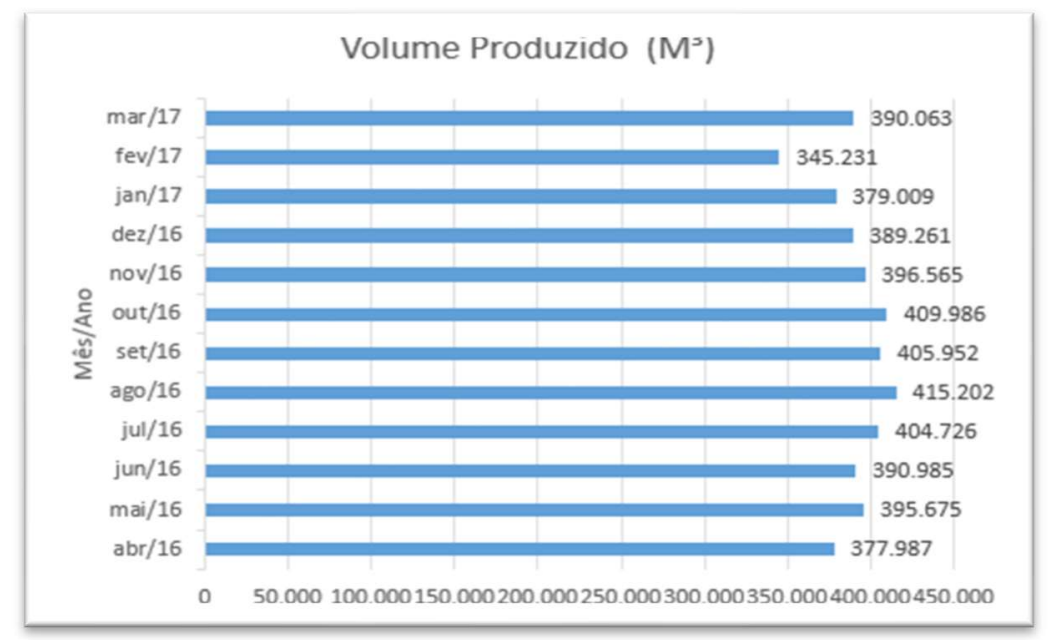

Figura 3: Gráfico do Volume Distribuído em Porto Nacional. 
Fazendo a análise do gráfico acima, pode-se notar que o mês com maior volume disponibilizado é o de Agosto/2016, enquanto que o menor foi apresentado em Fev/2017.

\section{Volume utilizado (micromedido) e volume faturado}

No Quadro 2 abaixo, demostra o Volume Utilizado, e o Volume Faturado:

Quadro 2: Histórico dos Volumes Micromedidos e Faturados

\begin{tabular}{|c|c|c|c|}
\hline MÊS/ANO & LOCALIZAÇÃO & MICROMEDIDO (M³) & VOLUME FATURADO(Mํ) \\
\hline ABR/2016 & \multirow{12}{*}{ PORTO NACIONAL } & 191.720 & 228.103 \\
\hline $\mathrm{MAI} / 2016$ & & 200.653 & 234.954 \\
\hline JUN/2016 & & 205.543 & 236.899 \\
\hline JUL/2016 & & 207.326 & 233.842 \\
\hline AGO/2016 & & 205.289 & 240.787 \\
\hline SET/2016 & & 215.959 & 248.059 \\
\hline OUT/2016 & & 195.616 & 231.974 \\
\hline NOV/2016 & & 197.554 & 232.598 \\
\hline DEZ/2016 & & 199.365 & 234.083 \\
\hline JAN/2017 & & 212.431 & 240.998 \\
\hline FEV/2017 & & 176.468 & 220.211 \\
\hline MAR/2017 & & 178.976 & 220.626 \\
\hline
\end{tabular}

\section{Determinação dos principais indicadores de perdas}

Para a determinação dos indicadores de perdas, total de dias e o volume total de perdas, foram contados o intervalo de tempo entre os meses de Abri/2016 e Mar/2017. A pressão média do setor foi fornecida pela Companhia de saneamento BRK Ambiental, portanto, foram considerados: Volume perdido

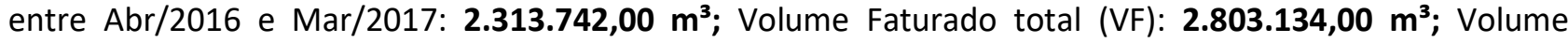
Micromedido total (VM): 2.386.900,00 m³; Número de dias entre fevereiro e agosto: 365 dias; Pressão média:

\section{2 m.c.a}

Dentro do período fornecido, obtém-se o volume perdido, que resulta do valor disponibilizado, menos o volume micromedido.

\section{Índice de Perda na Distribuição (IPD) ou Água Não Contabilizada (ANC)}

Com auxílio das informações disponibilizados pela concessionária, o índice de perdas totais na distribuição de Porto Nacional, foi obtido através do indicador percentual de perdas. O volume mensal de perdas e o índice de perdas totais do município estão representados pelas Figuras 4 e 5.

Durante o período analisado, o volume perdido foi de $2.313 .742,00 \mathrm{~m}^{3}$, porém para atingir o valor máximo considerado bom segundo a literatura, o volume deveria ser de $1.175 .381 \mathrm{~m}^{3}$. A média percentual de perdas encontrada no período disponibilizado foi de $49,2 \%$, sendo essa média considerada ruim para a cidade de Porto Nacional/TO. 

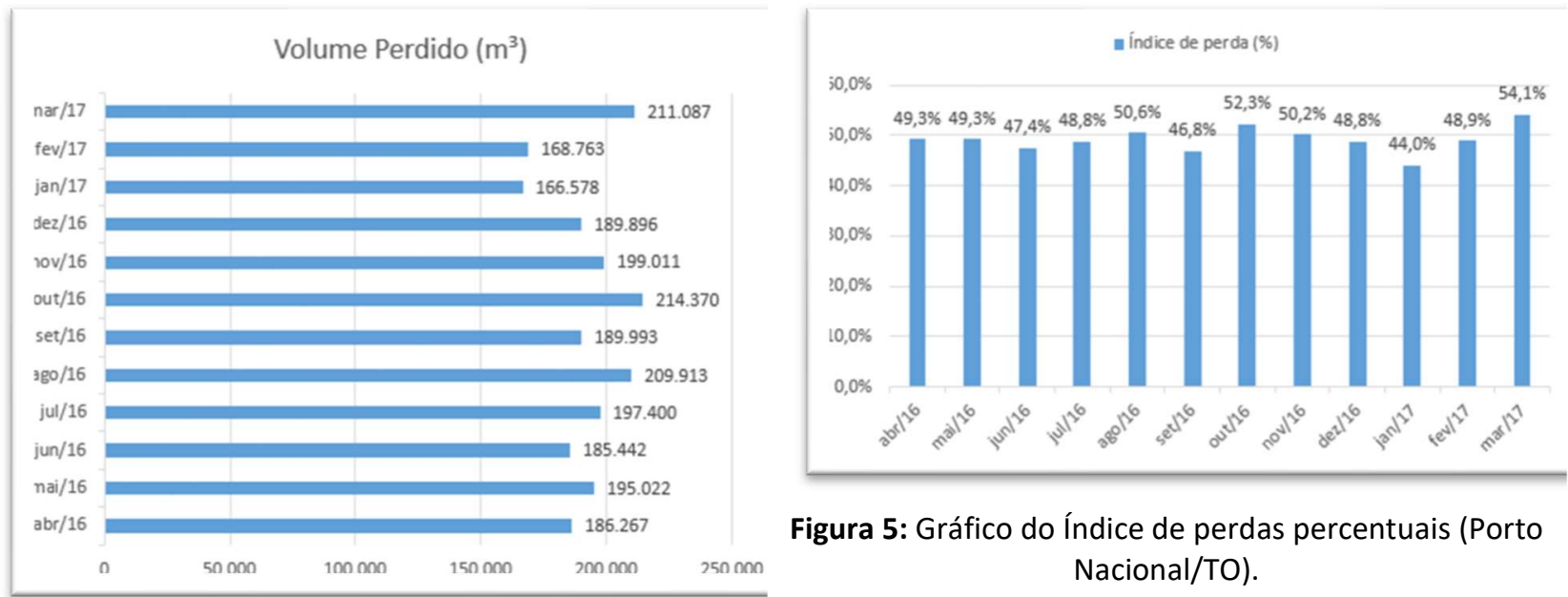

Figura 5: Gráfico do Índice de perdas percentuais (Porto Nacional/TO).

Figura 4: Gráfico do Volume Perdido em Porto Nacional.

Através cálculos realizados, o Quadro 3 demostra o número de ligações ativas, o número de dias para cada mês e o valor do Indicador de perdas por ramal dos meses analisados, além do volume perdido. A média do IPR foi de 350,62 L/ramal.dia, com desvio padrão de 33,72 L/ramal.dia. Porém os meses de Ago/2016, Out/2016, Jan/2017 e Fev/2017 constataram valores maiores e/ ou que a do desvio padrão.

Quadro 3: Indicador de Perdas por Ramal (IPR).

\begin{tabular}{|l|l|l|l|l|}
\hline MÊS/ANO & No DE DIAS & VOLUME PERDIDO $\mathbf{~}^{\mathbf{3}}$ ) & LIG. ATIVAS & IPR (L/RAMAL.DIA) \\
\hline ABR/2016 & 30 & 186.267 & 16.383 & 341,09 \\
\hline MAI/2016 & 31 & 195.022 & 16.459 & 367,32 \\
\hline JUN/2016 & 30 & 185.442 & 16.525 & 336,66 \\
\hline JUL/2016 & 31 & 197.400 & 16.584 & 368,99 \\
\hline AGO/2016 & 31 & 209.913 & 16.650 & 390,83 \\
\hline SET/2016 & 30 & 189.993 & 16.708 & 341,14 \\
\hline OUT/2016 & 31 & 214.370 & 16.759 & 396,53 \\
\hline NOV/2016 & 30 & 199.011 & 16.814 & 355,08 \\
\hline DEZ/2016 & 31 & 189.896 & 16.921 & 347,90 \\
\hline JAN/2017 & 31 & 166.578 & 16.988 & 303,97 \\
\hline FEV/2017 & 28 & 168.763 & 17.079 & 276,68 \\
\hline MAR/2017 & 31 & 211.087 & 17.164 & 381,25 \\
\hline Média & 30 & 192.812 & 16.753 & 350,62 \\
\hline
\end{tabular}

Em razão do número de ligações ativas e o volume micromedido, foi elaborada o Quadro 4, onde foi calculado o volume de água utilizado por ligação por dia (em L/lig.dia).

Quadro 4: Indicador de Perdas por Ramal (IPR).

\begin{tabular}{|l|l|l|l|l|}
\hline MÊS/ANO & No DE DIAS & VOLUME MICROMEDIDO (M $\left.\mathbf{3}^{\mathbf{3}}\right)$ & LIG. ATIVAS & IPR (L/LIG.DIA) \\
\hline ABR/2016 & 30 & 191.720 & 16.383 & 351,07 \\
\hline MAI/2016 & 31 & 200.653 & 16.459 & 377,92 \\
\hline JUN/2016 & 30 & 205.543 & 16.525 & 373,15 \\
\hline JUL/2016 & 31 & 207.326 & 16.584 & 387,55 \\
\hline AGO/2016 & 31 & 205.289 & 16.650 & 382,22 \\
\hline SET/2016 & 30 & 215.959 & 16.708 & 387,76 \\
\hline OUT/2016 & 31 & 195.616 & 16.759 & 361,84 \\
\hline NOV/2016 & 30 & 197.554 & 16.814 & 352,48 \\
\hline DEZ/2016 & 31 & 199.365 & 16.921 & 365,25 \\
\hline JAN/2017 & 31 & 212.431 & 16.988 & 387,65 \\
\hline
\end{tabular}




\begin{tabular}{|l|l|l|l|l|}
\hline FEV/2017 & 28 & 176.468 & 17.079 & 289,31 \\
\hline MAR/2017 & 31 & 178.976 & 17.164 & 323,25 \\
\hline MÉDIA & 30 & 198.908 & 16.753 & 361,62 \\
\hline
\end{tabular}

Todos os meses apresentaram um valor muito próximo da média de 361,62 litros por ligação por dia.

\section{Indicador de Perda de Faturamento (IPF) ou Água Não Faturada (ANF)}

O Quadro 5 mostra o índice de perda de faturamento dos meses em estudo:

Quadro 5: Índice de Perdas de Faturamento (IPF).

\begin{tabular}{|l|l|l|l|}
\hline MÊS/ANO & MICROMEDIDO $\left(\mathbf{M}^{\mathbf{3}}\right)$ & VOLUME FATURADO(M $\left.\mathbf{3}^{\mathbf{3}}\right)$ & IPF(\%) \\
\hline ABR/2016 & 377.987 & 228.103 & 39,65 \\
\hline MAI/2016 & 395.675 & 234.954 & 40,62 \\
\hline JUN/2016 & 390.985 & 236.899 & 39,41 \\
\hline JUL/2016 & 404.726 & 233.842 & 42,22 \\
\hline AGO/2016 & 415.202 & 240.787 & 42,01 \\
\hline SET/2016 & 405.952 & 248.059 & 38,89 \\
\hline OUT/2016 & 409.986 & 231.974 & 43,42 \\
\hline NOV/2016 & 396.565 & 232.598 & 41,35 \\
\hline DEZ/2016 & 389.261 & 234.083 & 39,86 \\
\hline SAN/2017 & 379.009 & 240.998 & 36,41 \\
\hline FEV/2017 & 345.231 & 220.211 & 36,21 \\
\hline MAR/2017 & 390.063 & 220.626 & 43,44 \\
\hline MÉDIA & 391.720 & 233.595 & 40,29 \\
\hline
\end{tabular}

Dada a média do IPF foi de 40,29 \%, e o desvio padrão de 2,28\%, todos meses acima analisadas estão de acordo com o padrão nacional, que segundo a literatura deve estar entre as taxas de $25 \%$ e $65 \%$.

\section{DISCUSSÃO}

O volume de perdas que existem em um sistema de abastecimento de água, pode ser encontrado desde a residência onde a água chega ao consumidor até a sua capitação, todavia esse processo se encontra ativo em praticamente todos os sistemas de tratamento da água existentes nas cidades brasileiras. Devido a isso, o custo para operação desses sistemas acaba gerando um elevado índice de prejuízos no faturamento (SANTOS et al., 2014).

Segundo Miranda (2002), o elevado índice de perdas nas concessionárias e prestadoras de serviço ao abastecimento público, são gerados por fatores como: Capacidade Institucional e gerenciamento dos sistemas com baixo nível de gestão; Recursos limitados para investimentos em planos de melhor desenvolvimento tecnológico, tanto para operação dos sistemas e rede de distribuição; Baixa preocupação com uso e conservação de modo racional, gerando; Falta de estudos e concepção de projetos, à medida que surgem necessidades e ampliação da carga hidráulica e extensão de redes para áreas mais distantes do sistema.

De uma forma simples, o conceito de perdas é representado pela diferença entre a água tratada disponibilizada pela concessionária (macromedição), e os valores medidos no hidrômetro de consumidor (micromedição). Andrade Sobrinho et al. (2016) apontam para a elaboração de um método que favoreça o controle de perdas, esse plano é chamado de check-list e se baseia nos problemas como: qual o volume de 
água perdido? Em que lugar a água está sendo perdida? Como aumentar a performance do sistema? Como conservar o funcionamento do sistema em alta?.

Para uma maior eficiência que garanta o controle de perdas, deve ser implantado um modelo de gerenciamento rotineiro, sobre o processo de operação de sistemas de abastecimento de água, bloqueio das causas que geram perdas, ações de conscientização da população e sensibilização com intuito de promover uma maior consciência sobre a problemática da existente (BAGGIO, 2000).

\section{CONCLUSÕES}

Dentre o intervalo de Tempo entre Abril de 2016 e Março de 2017, obteve-se para o quadro de perdas uma média percentual de $49,2 \%$, sendo que para alcança esse valor, foi levado em consideração os volumes disponibilizados e micromedido. Ao conseguir o valor percentual de perdas, observa-se que o índice de desperdício nesse período de tempo se encontra muito elevado com reação aos valores conhecidos em bibliografia especializada.

Para garantir um melhor, desempenho no sistema por um todo da cidade de Porto nacional, devem ser feitas algumas melhorias nas áreas de gestão comercial e operacional, sendo que uma melhora na gestão comercial, atribui numa eficiência no controle de fraudes e ligações clandestinas. Na parte operacional, um desenvolvimento das atividades em campo pode proporcionar uma maior redução no índice de vazamentos em redes, ramais e cavaletes, fatores todos esses considerados os principais responsáveis pelo elevado índice de perdas.

Para haja um melhor entendimento da população sobre os problemas e riscos causados pelas fraudes e/ou ligações clandestinas, um movimento de conscientização pode ser apresentado, visto que a medida em que forem descobertas irregularidades causada por infratores, resulta em prejuízo alto para população cliente, que passam a sofrer ainda mais com o aumento das tarifas, proporcionais ao índice de perdas existentes e à medida em que ele aumenta.

Portanto, as vantagens adquiridas com um maior investimento na área de combate a perdas garantem benefícios, para a área empresarial, que aumentará a porcentagem de lucro e diminuirá no quantitativo de gasto e também para o consumidor, solucionando os valores de tarifas e dando maior eficiência em problemas relacionados a falta de água. Todavia, o meio ambiental também é beneficiado, sedo que evitando o desperdício, a população obtém uma maior disponibilidade de tempo a água tratada.

\section{REFERÊNCIAS}

ABES. Associação Brasileira de Engenharia Sanitária e Ambiental. Controle e redução de perdas nos sistemas públicos de abastecimento de água: posicionamento $\mathrm{e}$ contribuições técnicas da ABES. ABES, 2015.

ANDRADE SOBRINHO, R.; BORJA, P. C.. Gestão das perdas de água e energia em sistema de abastecimento de água da Embasa: um estudo dos fatores intervenientes na RMS. Engenharia Sanitária e Ambiental, v.21, n.4, p.783795, 2016. DOI: https://doi.org/10.1590/s1413$\underline{41522016116037}$
BAGGIO, M. A.. Diagnóstico de perdas de sistemas de abastecimento de água. Franca: ABES, 2000.

TRATA BRASIL. Perdas de água 2018 (SNIS 2016): desafios para disponibilidade hídrica e avanço da eficiência do saneamento básico. São Paulo: TRATA BRASIL, 2019.

CREDER, H.. Instalações hidráulicas e sanitárias: Livros Técnicos e Científicos. 6 ed. Rio de Janeiro: LTC, 2006. 
SOUSA, E. R.. Noções sobre qualidade da água. Monografia (Licenciatura em Engenharia Civil) - Instituto Superior Técnico, Lisboa, 2001.

GONÇALVES, D. F. H.. Análise das perdas de água do sistema de abastecimento do Município de Pomerode/SC. Monografia (Bacharelado em Engenharia Sanitária e Ambiental) - Universidade Federal de Santa Catarina, Santa Catarina, 2018.

MIRANDA, E. C.. Avaliação de perdas em sistemas de abastecimento de água: indicadores de perdas e metodologias para análise de confiabilidade. Dissertação (Mestrado em Tecnologia Ambiental e Recursos Hídricos) Universidade de Brasília, Brasília, 2002.
SANTOS, D. D.; MONTENEGRO, S. M. G. L.. Avaliação da metodologia para controle de perdas de água em rede de distribuição no Recife-PE. Revista DAE, Recife, v.197, n.1, p.56-80, 2014. DOI: http://dx.doi.org/10.4322/dae.2014.128

TSUTIYA, M. T.. Abastecimento de Água. 2 ed. São Paulo: Departamento de Engenharia Hidráulica e Sanitária da Escola Politécnica da Universidade de São Paulo, 2005.

WERDINE, D.. Perdas de água em sistemas de abastecimento. Dissertação (Mestrado em Engenharia da Energia) - Universidade Federal de Itajubá, Itajubá, 2002.

A CBPC - Companhia Brasileira de Produção Científica (CNPJ: 11.221.422/0001-03) detém os direitos materiais desta publicação. Os direitos referem-se à publicação do trabalho em qualquer parte do mundo, incluindo os direitos às renovações, expansões e disseminações da contribuição, bem como outros direitos subsidiários. Todos os trabalhos publicados eletronicamente poderão posteriormente ser publicados em coletâneas impressas sob coordenação da Sustenere Publishing, da Companhia Brasileira de Produção Científica e seus parceiros autorizados. Os (as) autores (as) preservam os direitos autorais, mas não têm permissão para a publicação da contribuição em outro meio, impresso ou digital, em português ou em tradução. 